\title{
BMJ Open Protocol for determining primary healthcare practice characteristics, models of practice and patient accessibility using an exploratory census survey with linkage to administrative data in Nova Scotia, Canada
}

\author{
Emily Gard Marshall, ${ }^{1}$ Richard J Gibson, ${ }^{2}$ Beverley Lawson, ${ }^{1}$ Frederick Burge ${ }^{1}$
}

To cite: Marshall EG, Gibson RJ, Lawson B, et al. Protocol for determining primary healthcare practice characteristics, models of practice and patient accessibility using an exploratory census survey with linkage to administrative data in Nova Scotia, Canada. BMJ Open 2017;7:e014631. doi:10.1136/bmjopen-2016014631

- Prepublication history and additional material is available. To view please visit the journal (http://dx.doi.org/ 10.1136/bmjopen-2016014631).

Received 11 October 2016 Revised 24 January 2017 Accepted 20 February 2017

CrossMark

\footnotetext{
${ }^{1}$ Department of Family Medicine, Dalhousie University, Halifax, Nova Scotia, Canada ${ }^{2}$ Department of Family Practice, Nova Scotia Health Authority, Halifax, Nova Scotia, Canada
}

Correspondence to Dr Emily Gard Marshall; emily.marshall@dal.ca

\section{ABSTRACT}

Introduction: There is little evidence on how primary care providers (PCPs) model their practices in Nova Scotia (NS), Canada, what services they offer or what accessibility is like for the average patient. This study will create a database of all family physicians and primary healthcare nurse practitioners in NS, including information about accessibility and the model of care in which they practice, and will link the survey data to administrative health databases.

Methods and analysis: 3 census surveys of all family physicians, primary care nurse practitioners (ie, PCPs) and their practices in NS will be conducted. The first will be a telephone survey conducted during typical daytime business hours. At each practice, the person answering the telephone will be asked questions about the practice's accessibility and model of care. The second will be a telephone survey conducted after typical daytime business hours to determine what out-of-office services PCP practices offer their patients. The final will be a tailored fax survey that will collect information that could not be obtained in the first 2 surveys plus new information on scope of practice, practice model and willingness to participate in research. Survey data will be linked with billing data from administrative health databases. Multivariate regression analysis will be employed to assess whether access and availability outcome variables are associated with PCP and model of practice characteristics. Negative binomial regression analysis will be employed to assess the association between independent variables from the survey data and health system use outcomes from administrative data.

Ethics and dissemination: This study has received ethical approval from the Nova Scotia Health Authority and the Health Data Nova Scotia Data Access Committee. Dissemination approached will include stakeholder engagement at local and national levels, conference presentations, peer-reviewed publications and a public website.

\section{Strengths and limitations of this study}

- This is the first census study in Canada to systematically capture practice and provider characteristics and link them to administrative data to answer questions about models of primary healthcare and healthcare access, comprehensiveness and utilisation.

- This study was developed in collaboration with local knowledge users in response to an identified priority area for data and research, with contributions to tool development and ensuring the relevance of the research to policy priorities.

- This study will use multiple methods to collect data from practices and providers, including sources that do not only rely on directly surveying physicians to lessen the burden on providers' time, and when required, using tailored methods to improve physician response rates. Multiple methods allow the establishment of reliability between data sources and to triangulate study findings.

- Limited coding structures for primary care providers and inconsistencies in data entry may present limitations in the data available in the administrative databases.

- Response rates for surveys of physicians are historically low; this study will mitigate this limitation with robust recruitment strategies and data collection plan, minimising physician and nurse practitioner time.

\section{INTRODUCTION}

Access to and quality of primary healthcare (PHC), including models of collaborative and team-based care, are key areas of interest for policy and research. Accessible, wellcoordinated team-based PHC is associated with better health outcomes and lower healthcare system costs. ${ }^{1-6}$ In Nova Scotia (NS), improved access to PHC and the 
development of interprofessional teams are major strategic goals of the provincial Department of Health and Wellness and the Nova Scotia Health Authority (NSHA) ${ }^{78}$ However, to date, there is very little empirical evidence on how primary care providers (PCPs) model their primary care practices in NS, what services they offer or what accessibility is like for the average patient. This innovative Models and Access Atlas of Primary Care in Nova Scotia (MAAP-NS) study will create a database, or atlas, of all family physicians and PHC nurse practitioners (ie, PCPs) in NS, accessibility to their services, models of care and PCP characteristics. The second phase of MAAP-NS will link the survey data 'atlas' of all PCPs to administrative health databases. This will allow for exploration of research issues such as: the association of models of primary care with chronic disease prevention and management; accessibility of primary care with patient use of emergency departments; and walk-in clinic utilisation and preventive and screening services. This research will: (1) identify areas of PHC requiring enhancement, such as where gaps exist in accessibility (eg, geographical location, time of day and availability of specific primary care services); (2) provide a baseline for evaluating future innovations and (3) create new knowledge about the current landscape of PHC access, models of care being offered and their impacts on utilisation.

\section{Access to PHC}

The vast majority of medical care in NS is primary care provided by family physicians and nurse practitioners. Of the $\sim 4.1$ million medical encounters each year in NS (primary care visits, emergency room visits, surgeries and hospital admissions), $80 \%$ are to family physicians and nurse practitioners. ${ }^{9}$ It is well documented that accessible and well-coordinated primary care is associated with better health outcomes and lower costs to the healthcare system. ${ }^{1}{ }^{3}{ }^{5}$ Adults with an accessible source of primary care are more likely to receive preventive care and experience fewer disparities in care when compared with those without a source of care. ${ }^{10}$

However, having a PCP does not ensure access; in one study, the majority of participants reported having a PCP but most of those reported having difficulties accessing care. ${ }^{11}$ In large surveys of Canadians in 2007 and 2008, $84-91 \%$ of participants reported having a PCP. ${ }^{312}$ The most recent data indicate that $85.1 \%$ of Canadians have a regular PCP. ${ }^{13}$ Yet, the percentage of Canadians reporting unmet healthcare needs has increased from 1995 $(4.2 \%)$ to $2014(11.2 \%) .{ }^{13}{ }^{14}$ Reasons for unmet healthcare needs include wait times, the geographic location of physicians' offices, the limited hours of operation at family physicians' offices and, broadly, that services were not available at the time they were required. ${ }^{12} 1415$

\section{Models of primary care practice}

Increasingly, new models of primary care have been implemented across Canada and in NS. An inventory and synthesis of new models of health services delivery reported on new, emerging and existing models of health services delivery in Canada and found that in NS, there are eight different models (primary and non-PHC) in practice, while in Ontario, there are $32 .{ }^{16}$ Similarly, the Health Council of Canada has outlined types of primary care teams currently operating in $\mathrm{NS}^{17}$ and in 2008, the Nova Scotia Department of Health and Wellness used the work of Pineault $e t a l^{18}$ to describe key features of various models of PHC practice, focusing on elements of accessibility, continuity of care, number of PCPs and other allied health professionals involved in the practice, and collaboration and integration.

Currently, the NSHA is using the following classification of primary care models in their work: (1) Solo Family Physician Practice; (2) Group Practice (an organisation of two or more family physicians who work together, share client/patient records, office space, staff, technology and on-call coverage); (3) Interdisciplinary Healthcare Team or Network (includes a group of individuals with diverse training who work together to deliver patient care, such as family physicians, nurses, social workers, dieticians and other healthcare providers) and (4) Other. However, these model frameworks are not a comprehensive list of what models of care are present in NS and there are still several unknowns regarding models of and access to primary care in NS, including: if the listed classifications are sensitive enough to capture the reality of practice offerings in NS; how many physicians are practicing in each mode and what components of access are correlated with each model. ${ }^{17-19}$

\section{Study context and objectives}

To ensure the feasibility of obtaining response rates from PCPs, we first conducted a pilot-feasibility study. In the pilot phase, we built an interprofessional research team, including healthcare researchers, family physicians, nurse practitioners and primary care representatives from the provincial Department of Health and Wellness and NSHA; built relationships with our stakeholder groups; and refined our data collection tools, which will be used in the full study. The survey tools were developed from an extensive search of existing tools, including the Canadian Institute of Health Information Primary Health Care Indicators Chartbook. ${ }^{15}$ Our team strategised methods to obtain data with minimal reliance on PCP responses and with maximised PCP response rates, where required. This included maximising data collection from non-PCP sources. These sources include information publicly available from the College of Physician and Surgeons website; a list of PCPs' names, contact information, training and specialty from the Nova Scotia Department of Health and Wellness; and conducing the first surveys by calling the PCP office and asking the person who answers the phone a number of questions. During this iterative pilot study phase, item responses were 
examined to determine if they could be shorter and if any required responses from the PCP directly. For example, forms of remuneration were often not known by office phone respondents. Team review of the survey tools and pilot responses led to the development of shorter, targeted surveys that make the most efficient use of PCP time. The full study will use these novel survey methods to investigate the current availability of and access to all PCPs in NS from the patient perspective plus provider and practice characteristics. The objectives of this study are:

1. To create an atlas of all known actively practicing family physicians and nurse practitioners in NS with key characteristics (ie, location, gender, provider identification number, scope of practice and willingness to participate in future research).

2. To determine the models of primary care practice in which all family physicians and nurse practitioners in NS work (ie, solo physician, group practice or interdisciplinary team; disciplines included on primary care teams).

3. To assess access to and availability of all family physicians and nurse practitioners in NS (ie, telephone and email access, message service, office hours, out-of-hours arrangements, accepting new patients, next available appointment, same-day appointments) and examine the relationship to PCP key characteristics and the model of primary care in which they practice.

4. To examine the association of key PCP and practice characteristics (such as location, gender, models of primary care practice, accessibility) and patient health service utilisation outcomes (such as emergency department and walk-in clinic use; prevention and screening services), using information captured in provincial administrative health data.

\section{METHODS AND ANALYSIS}

\section{Survey data collection}

The survey data collection phase of the study will involve three population surveys: two telephone surveys and one fax survey. For all three surveys, information supplied by the Department of Health and Wellness of NS will be used to contact all primary care practices and providers in NS (projected population size $=828$ PCPs). Since all three surveys will be complete censuses of the population of PCPs, no sample size calculations are required.

The first survey is a telephone survey of all primary care practices and will be conducted during typical daytime business hours. PCP offices will be notified about the study with a letter of information that demonstrates the significance of the research and the team's direct links to policymakers. Each office phone number will be called by a trained research assistant a maximum of five times on different days of the week at different times of the day to maximise the opportunity to reach the practice. If offices are unreachable after five attempts, a personalised letter will be sent to the PCP explaining the study, that their office was unreachable despite five attempts on different days of the week and that they are invited to contact the study by email if they want to arrange participation. At each practice, the person answering the telephone will be asked about whether the practice is accepting new patients, the hours of operation, the next available patient appointment for routine care or for urgent care and whether same-day appointments are offered. Information about the structure of the practice in terms of number of providers, presence of interdisciplinary professionals, sharing of resources and the presence of on-call and after-hours care will also be collected (see online supplementary appendix A). For group practices, data will be collected for each individual PCP working in the practice. Colocation and collaboration indicators will be used to determine group practices versus colocated independent PCPs.

Similarly, the second survey will be a telephone survey of all primary care practices but will be conducted after typical business hours. It will determine if practices provide, via recorded message or on-call service, information on out-of-hours arrangements for patients and what they are (see online supplementary appendix B).

The third survey is a fax survey of all PCPs in NS. We will use fax communication to reach providers directly as it reflects the communication technologies currently in use by PCPs in NS; we heard from PCPs in our pilot study that this was their preferred medium for the surveys as it fits directly into their work flow. The information sought will concern scope of practice and aspects of each provider's model of primary care and will include a request for consent to be contacted for future research (see online supplementary appendix $\mathrm{C}$ ). This survey will be tailored to each PCP to remove items that ask about information that was obtained in the first two surveys.

Detailed protocols and instructions for the provider and practice surveys will be used to train and guide research assistants (see online supplementary appendix D). Data collection began January 2014 and is ongoing, with an anticipated end date of June 2017. All data will be entered into spreadsheets and analysed with SPSS, V.22 (IBM Corp. Released 2013. IBM SPSS Statistics for Windows, V.22.0. Armonk, New York, USA: IBM Corp).

\section{Data linkage}

In Canada, PCPs deliver healthcare free of cost to the general public. To be remunerated, they bill the provincial government for their work using unique billing codes for each service provided to each patient. Some family physicians and nurse practitioners are paid using alternative methods, such as salaries, but they still submit 'shadow' billing information to the provincial government. These administrative billings databases document which medical services (including referrals to specialists and use of emergency departments) are provided to 
which patient by which provider. These databases also contain some patient information, such as diagnostic codes for chronic diseases and procedures delivered to patients. This study will not access the individual patient data, but will have access to patient data aggregated at the provider level.

Following the survey data collection, survey data will be linked with billing data from the administrative databases held by Health Data Nova Scotia (HDNS). The PCPs' individual billing numbers will be sent to Medavie Blue Cross, which maintains the Medical Services Insurance (MSI) database for NS, for encryption. Accompanying the billing numbers will be a unique identifier generated within the MAAP-NS data set. The encrypted billing numbers and MAAP-NS ID numbers will be sent to the Maritime SPOR Support Unit (MSSU). The encrypted billing numbers will allow extraction of billing data for the PCPs in the study and the MAAP-NS ID will allow linkage of the PCPs' survey data to their administrative billing data (see figure 1).

\section{Analysis plan}

Initial analyses to develop the atlas will be primarily descriptive. Frequencies, proportions, measures of central tendency and variance will be applied to all data. For example, mean age (with SD), proportion of men and women, mean time since graduation, proportion rural/urban, proportion of PCPs practicing in each model of primary care will be calculated, as well as access and availability frequencies, means and distributions. Next, bivariate analyses will be used to examine the relationship between key characteristics of actively practicing PCPs, model of primary care practice and accessibility (eg, mean time to next available nonurgent/urgent appointment in days by age of PCP, rural/urban characteristics or by model of primary care practice). Finally, multivariate regression analysis will be employed to assess whether access and availability outcome variables (eg, accepting new patients, wait times) are associated with physician and model of practice characteristics. For example, a logistic regression will be used to assess if primary care practice structures are associated with accepting new patients (yes vs no) after controlling for geographical location, number of hours available for patients per week, physician gender, age and years since graduation.

The development of indices of collaboration will involve factor analysis of the survey items related to collaborative elements. These include: the sharing of physical resources (waiting rooms, examination room and equipment); sharing of human resources (staff, on-call, coverage during absences); sharing of patients and records; and formal or informal practice protocols. Development of understanding of models of care will include examining combinations of constituent elements related to the descriptions of primary care models in the literature, including types and numbers of interprofessional care providers colocated, methods of collaboration, methods of remuneration and clinic governance. The various models and their constituent components will all be analysed to see if there is a relationship with key outcomes of interest, including availability, accessibility and comprehensiveness of services offered.

Billing data linkage will allow for analysis to see if provider characteristics, practice characteristics, aspects of collaboration and models of care predict healthcare utilisation patterns of patients, rates of using walk-in clinics and emergency department use, and whether patients are more likely to receive appropriate chronic disease prevention and management. See supplementary appendix $\mathrm{E}$ for the objectives and methods for the billing data linkage analysis.

Negative binomial regression analysis will be employed to assess the association between independent variables of interest such as models of primary care and accessibility and 'count' data, including the total number of visits to an emergency department, walk-in clinic, total ambulatory visits and total targeted services. Unadjusted regression will be followed by multivariate techniques to allow for the control of various patient, physician and practice factors that may modify the effect of the association. If a disproportionately large number of patients did not experience any emergency or walk-in visits (ie, count $=0$ ), these analyses will be repeated using logistic regression techniques to evaluate the probability of having made at least one visit versus none. To address the possibility of non-independence among cases (ie, that patients of one provider may be more similar to one another than they are to patients of another provider), we will attempt multilevel regression modelling. This procedure adds a variable to each regression model for which each provider has a value. This allows the inclusion of individuals and groups of individuals in the same model to avoid flouting the assumption of independent cases.

\section{DISSEMINATION}

All information received from participants will be treated in a confidential manner and stored in passwordprotected files on a secure research drive at Dalhousie University Department of Family Medicine, Primary Care Research Unit. The linked data set will be held and analysed in the high-security facilities of the MSSU.

Knowledge translation and exchange activities will be facilitated by numerous committees and working groups in which the research team members are actively engaged, including (1) the Canadian Association of Health Services and Policy Research (CAHSPR) Primary Healthcare Theme Working Group, which provides opportunities for engagement and sharing of knowledge with PHC decision makers and researchers from across Canada, (2) the Transdisciplinary Understandings and Training on Research-Primary Health Care (TUTOR-PHC) programme, which provides 


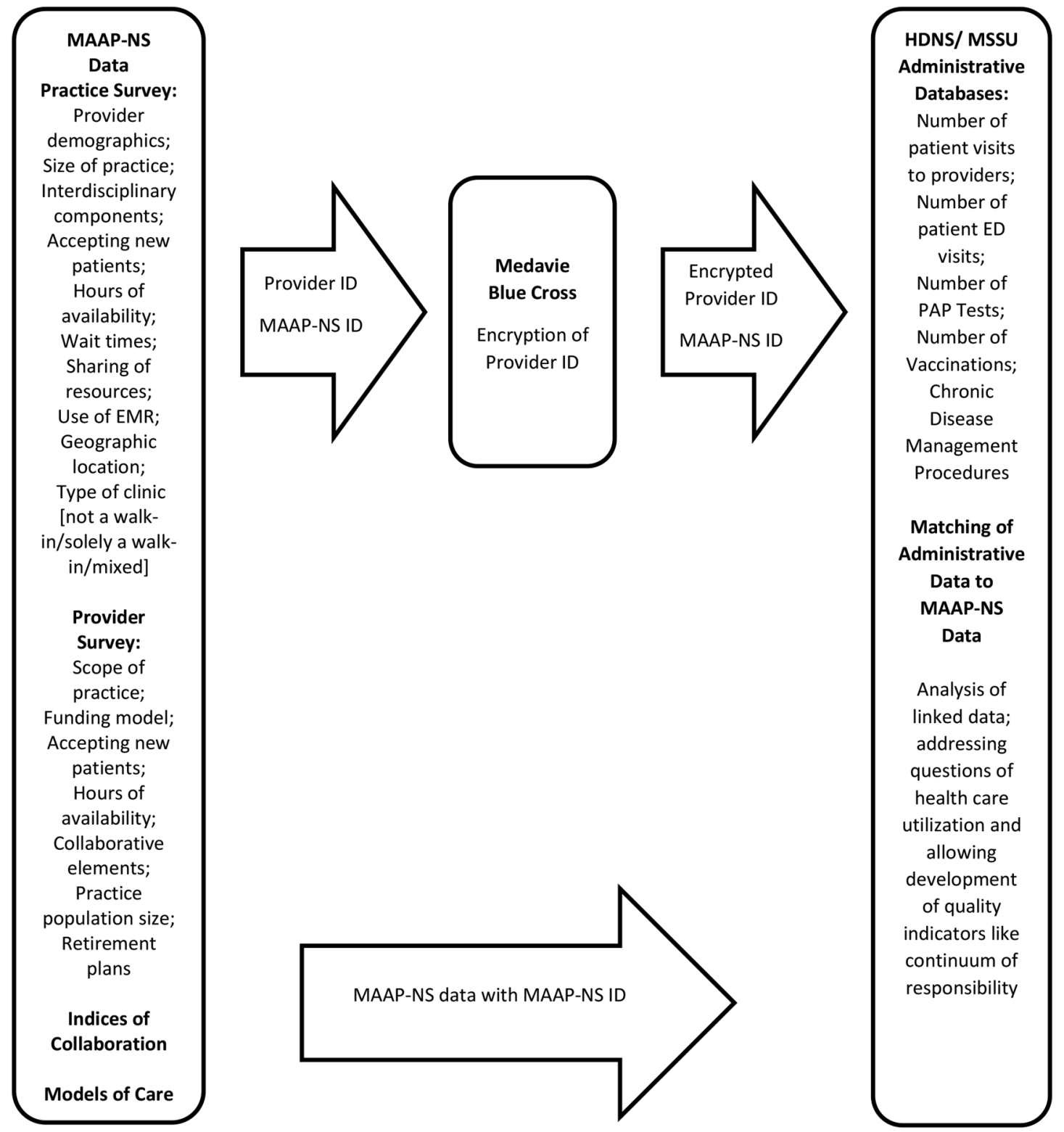

Figure 1 Data linkage in Models and Access Atlas of Primary Care-Nova Scotia.

interdisciplinary PHC research training and mentorship to a cohort of 12 graduate students from multiple disciplines from across the country annually and (3) the Canada Working Group on Primary Healthcare Improvement. As well as presentations at national and international meetings such as CAHSPR and North American Primary Care Research Group (NAPCRG), MAAP-NS findings will be made public on a website developed, especially for this study. The website will be maintained on the Dalhousie University server (http:// www.dal.ca/maapstudy) with up-to-date findings of the present study, as they become available, and will encourage visitors to provide feedback.

The overarching goal of this study is to provide evidence for models of primary care practice that lead to the highest level of access for the right care at the right time from the right provider. A provincial assessment of access is inline with a population health approach and will facilitate improving access to primary care to reduce growing health disparities. This study will generate a database (atlas) of PCPs in NS, accessibility to their services and models of care and will link this information to administrative databases. The atlas will be a valuable resource for answering future research and health policy questions. By establishing a database that contains relevant indicators of access to primary healthcare province-wide, it will be possible to determine what factors contribute to a more accessible primary care system, identify where gaps exist and where improvements can be made.

Funding This study is supported by an Establishment Grant from the Nova Scotia Health Research Foundation.

Competing interests None declared.

Ethics approval Research Ethics Board of the Nova Scotia Health Authority and from the Health Data Nova Scotia Data Access Committee. 
Provenance and peer review Not commissioned; externally peer reviewed.

Open Access This is an Open Access article distributed in accordance with the Creative Commons Attribution Non Commercial (CC BY-NC 4.0) license, which permits others to distribute, remix, adapt, build upon this work noncommercially, and license their derivative works on different terms, provided the original work is properly cited and the use is non-commercial. See: http:// creativecommons.org/licenses/by-nc/4.0/

\section{REFERENCES}

1. Starfield B, Shi L, Macinko J. Contribution of primary care to health systems and health. Milbank Q 2005;83:457-502.

2. Khan E, McIntosh C, Sanmartin C, et al. Working paper series: primary health care teams and their impact on process and outcomes for care (catalogue number 82-622-X no. 002). Ottawa, ON: Statistics Canada, 2008.

3. Schoen C, Osborn R, Doty MM, et al. Toward higher-performance health systems: adults' health care experiences in seven countries, 2007. Health Aff (Millwood) 2007;26:w717-34.

4. Russell GM, Dahrouge S, Hogg W, et al. Managing chronic disease in Ontario primary care: the impact of organizational factors. Ann Fam Med 2009;7:309-17.

5. Baicker K, Chandra A. Medicare spending, the physician workforce, and beneficiaries' quality of care. Health Aff (Millwood) 2004;4: w184-97.

6. Hollander MJ, Kadlec $\mathrm{H}$, Hamdi R, et al. Increasing value for money in the Canadian healthcare system: new findings on the contribution of primary care services. Healthc Q 2009;12:32-44.

7. Nova Scotia Health Authority. Nova Scotia Health Authority Business Plan. 2015. http://www.nshealth.ca/sites/nshealth.ca/files/ 2015-16_nsha_business_plan_-_master_final_2015-06-03.pdf

8. Capital District Health Authority. The Promise of Primary Health Care: The Community Master Plan Working Document. (n.d.). http:// www.cdha.nshealth.ca/system/files/sites/123/documents/ community-master-plan.pdf
9. Nova Scotia Department of Health and Wellness, Business Intelligence Analytics and Privacy Division. Retrieved 30 September 2016. http://www.nshealth.ca/about-us

10. Beal AC, Doty MM, Hernandez SE, et al. Closing the divide: how medical homes promote equity in health care. Results from the Commonwealth Fund 2006 Health Care Quality Survey, 2007. http:// www.commonwealthfund.org/usr_doc/1035_Beal_closing_divide _ medical_homes.pdf?section $=4039$

11. Wellstood K, Wilson K, Eyles J. 'Reasonable access' to primary care: assessing the role of individual and system characteristics. Health Place 2006;12:121-30.

12. Canadian Institute for Health Information. Analysis in Brief. Experiences with primary health care in Canada, July 2009. http:// secure.cihi.ca/cihiweb/products/cse_phc_aib_en.pdf

13. Statistics Canada. Unmet health care needs, 2014. Health Fact Sheets. Statistics catalogue no. 82-625-X. 2016. Retrieved 30 September 2016 http://www.statcan.gc.ca/pub/82-625-x/2016001/ article/14310-eng.htm

14. Sanmartin C, Houle C, Tremblay S, et al. Changes in unmet health care needs. Health Rep 2002;13:15-21.

15. Canadian Institute for Health Information (CIHI). Primary health care (PHC) Indicators chartbook: an illustrative example of using PHC data for indicator reporting. Ottawa, ON: CIHI, 2008. https://secure. cihi.ca/estore/productSeries.htm?pc=PCC426

16. Hollander Analytical Services Ltd. Assessing new models for the delivery of medical services: inventory and synthesis. August 2003. http://www.physicianhr.ca/reports/hollander-e.pdf

17. Health Council of Canada. Primary health care teams-a Nova Scotia perspective. 2009. http://www.healthcouncilcanada.ca/docs/ rpts/2009/TeamsInAction_NovaScotia_Summary.pdf

18. Pineault R, Levesque JF, Roberge D, et al. Primary care services organizational models and the population's care experience, Government du Quebec, Centre de recherché de l'hopital Charles LeMoyne. September 2008. http://www.greas.ca/evolution/pdf/843 modeles_organisation_ang.pdf

19. Health Council of Canada. Teams in action: primary health care teams for Canadians. 2009. http://www.dsrf.org/media/teamsinaction.pdf 